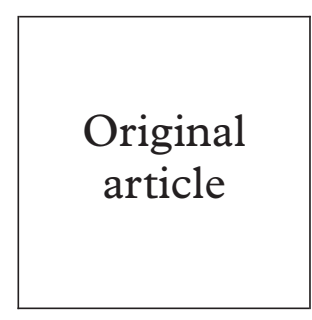

General Hospital St Georg, Hamburg,

Germany

A Plettenberg

D Albrecht

T Lorenzen

V Paech

T Weitzel

A Stoehr

Labor Fenner \& Partner, Hamburg, Germany

H Petersen

$\mathrm{T}$ Fenner

Labor Keeser/Arndt \& Partner, Hamburg, Germany

T Meyer

R Arndt

Virco NV Mechelen, Belgium

K Hertogs

Tibotec NV, Mechelen, Belgium

R Pauwels

Correspondence to: Andreas Plettenberg, MD, General Hospital St Georg, Infectious Diseases

Outpatient Department,

Haus Z, Lohmuehlenstrasse

5, D-20099 Hamburg,

Germany

plettenberg@

ifi-infektiologie.de

Accepted for publication 21 August 2001

\title{
Resistance analyses in HIV infected patients with a history of multiple antiretroviral treatment regimens
}

Andreas Plettenberg, Dirk Albrecht, Thore Lorenzen, Volker Paech, Heiko Petersen, Thomas Fenner, Thomas Meyer, Rüdiger Arndt, Kurt Hertogs, Rudi Pauwels, Thomas Weitzel, Albrecht Stoehr

Objective: To assess HIV-1 isolate based resistance profiles from extensively pretreated patients and effects of a resistance guided switch of antiretroviral therapy.

Methods: In a prospective study phenotypic and genotypic resistance analyses were performed on HIV infected individuals with failure of the current therapy and history of at least three antiretroviral regimens. Antiretroviral therapy was changed according to the results. Viral load and CD4 lymphocyte counts were measured at baseline, after 10 (SD 2), and 24 (2) weeks.

Results: All patients $(n=52)$ failed their actual regimen. Currently versus ever previously taking the specific drug, resistance associated mutations and phenotypic resistance to AZT and 3TC were found in over $80 \%$ of individuals; resistance to DDI and D4T was detected in less than $10 \%$ of cases. A resistance guided switch of therapy was followed by a median decrease of viral load of $0.5 \log 10$ units after 24 weeks. Individuals resistant to two or more drugs compared with patients with resistance to less than two drugs of ongoing treatment, were switched to a regimen containing DDI, D4T, and a PI or NNRTI. After 10 (SD 2) weeks viral load decrease was pronounced in patients with resistance to at least two drugs in the previous regimen.

Conclusions: Among different RTI, the profile of clinically relevant resistance indicates pronounced differences when looking at separate drugs. Regarding virological response, in the context of available drugs, resistance tested with currently used methods is of limited value in extensively pretreated patients and seems to have its value primarily in first or second switch of therapy.

(Sex Transm Inf 2001;77:449-452)

Keywords: HIV drug resistance; antiretroviral therapy; HIV diagnostic tests

\section{Introduction}

Advances in antiretroviral therapy (ART) have led to a dramatic decrease of morbidity and mortality in HIV positive patients, ${ }^{1}$ but in studies and in everyday clinical practice viral rebounds are observed. The frequency of this phenomenon increases with duration of treatment. ${ }^{23}$ Recently some studies were published reporting clinical benefits from genotypic and phenotypic resistance testing ${ }^{4-7}$; however, many important questions in everyday clinical management remain unconsidered. Performing resistance tests is a routine procedure in study settings while in everyday clinical life cost and insurance issues can cause significant delay. This evaluation was performed to learn more about relevance of genotypic and phenotypic resistance tests in extensively pretreated patients under everyday conditions.

\section{Materials and methods}

Data were collected from HIV positive patients in outpatient clinics of the General Hospital St Georg in Hamburg, Germany, between September 1997 and December 1998. Resistance testing was performed after failure of at least three different regimens of ART before resistance analysis. Viral load (Amplicor Test, Hoffmann LaRoche) and CD4 lymphocyte count were determined at the time antiretroviral therapy was changed after resistance testing, as well as 10 (SD 2) and 24 (2) weeks afterwards. For genotypic analysis of resistance associated mutations, the Amplicor HIV-1 monitor test (Roche Diagnostics) ultrasensitive protocol was used. Each gene (RT and PR gene) was cycle sequenced in forward and reverse direction and the raw data were analysed and edited by Sequence Navigator Software (Perkin Elmer, Weiterstadt, Germany). To identify relevant mutations for drug resistance, forward and reverse sequence of the RT and PR gene were aligned versus the HIV-1 reference strain pNL4-3, edited and loaded into the Complign module of the Mac Molly Tetra Software for further mutation analysis. All resistance associated mutations were reinterpreted using HIV-1 resistance mutation compilation (December 1998 version). ${ }^{8}$ Phenotyping drug resistance testing was performed using the recombinant virus technology (Antivirogram, Virco, Belgium) according to the method previously described. ${ }^{9}$

\section{Results}

BASELINE CHARACTERISTICS

A total of 52 HIV positive patients ( 47 males, five females) were analysed between July 1997 and March 1999 (median age 44 years; range 29-76 years). According to CDC classification, $73 \%$ fulfilled group C criteria, $19 \%$ group B, and $8 \%$ group A. A median of CD4 lymphocytes was 173 cells $\times 10^{6} / 1$ (range $3-470$ 

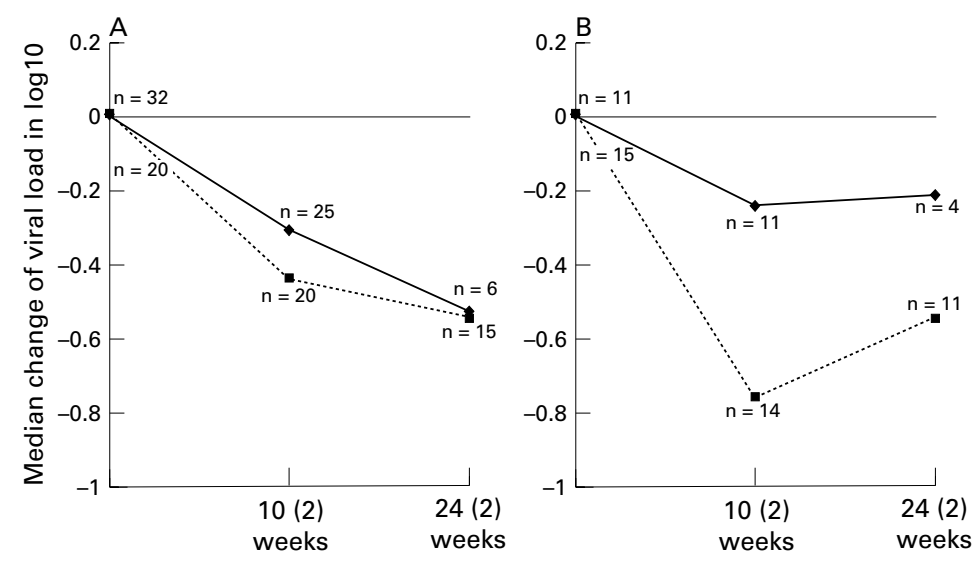

Increased in number of
susceptible drugs
in new regimen $-<2$
compared to old: $\cdots . . \geq 2$

Figure 1 (A) Virological outcome of change of therapy. The patients were observed in two groups according to the number of drugs in the new regimen to which their isolates were susceptible: group 1 with isolates susceptible to $\geqslant 2$ drugs versus group 2 including subjects with isolates susceptible to $<2$ drugs. Virological outcome of change of therapy for patients sensitive to ddI, d4T, and a PI or an NNRTI. The subjects were evaluated in two groups according to the number of drugs in the previous regimen to which isolates were resistant: group 1 with isolates resistant to $\geqslant 2$ drugs compared to group 2 with isolates resistant to $<2$ drugs.

cells $\left.\times 10^{6} / 1\right)$. ART had been changed in all patients at least three times before resistance testing. The median number of changes during therapy was six (three to nine) with median duration of ART before resistance analysis of 30.5 months (13-75 months).

Viral load change was separately observed for patients who were able to add at least two susceptible drugs, according to genotypic or phenotypic test in new therapy $(n=20)$, versus patients with one or less new susceptible drugs ( $\mathrm{n}=24)$; however, for both groups a median decrease of viral load of $0.5 \log 10$ was observed after 10 (SD 2) and 24 (2) weeks (fig 1A). For phenotypic tests a more than 10 -fold resistance was defined as relevant. Fourfold to 10 -fold resistance, frequently known as "intermediate resistance," was regarded as "susceptible" in this evaluation. In the combined analyses resistance was assumed when apparent in at least one system.

AVAILABLE DATA

Complete resistance testing (phenotypic and genotypic analysis) was performed in 38 of 52 patients $(73 \%)$, phenotypic testing only was available for six patients, and genotypic analysis only for eight patients.

RESISTANCE WITH REGARD TO CURRENTLY OR PREVIOUSLY TAKEN DRUGS

Firstly, we analysed resistance against drugs that were taken at time the sample was drawn; this resistance actually gives rise to virus replication in the respective patient. The second analysis was done to determine resistance to all drugs ever previously taken by the patient, representing cross resistance and/or "leftover" resistance induced by previous regimens. In one of 51 patients no phenotypic resistance to any ongoing drug was seen nor were resistance associated mutations detected in the viral isolate. In 15 patients resistance (assessed by phenotypic test and/or genotypic analysis) against one of the ongoing drugs was found. Thirteen patients with resistance against two current drugs, 16 individuals resistant against three, and six patients against four drugs were detected. Multiple drug resistance appeared common: 41 of 52 patients $(79 \%)$ had resistance against two or more RTIs (nucleoside analogue inhibitors of reverse transcriptase) whereas 28 of 52 (54\%) PI (protease inhibitor) treated individuals were found to be resistant against two or more drugs (table 1). Looking at single drugs, the percentage of patients with phenotypic resistance and/or significant resistance associated mutations against a certain drug was calculated for all patients taking this drug (open bars in fig 2) and for all patients ever on this drug (solid bars in fig 2). The median duration of intake and the number of patients available for each analysis of the respective drug is shown in the bottom half of figure 2. For patients actually on the respective drug as well as for patients ever treated with this drug, detectable resistance against RTIs was above $80 \%$ for AZT (zidovudine) and 3TC (lamivudine), but not more than $10 \%$ for DDI (didanosine), DDC (zalcitabine), and D4T (stavudine) in both types of analysis. With respect to the first analysis (current medication), the number of patients was highest for $3 T C(n=47)$ and was found to be highest for AZT $(n=50)$ in the second analysis. Duration of intake was longest for AZT (median 30 and 29 months) followed by 3TC (16 months) in both analyses. Identical analysis of single PIs showed less pronounced differences between the drugs. Most frequently, resistance was detected against ritonavir (RTV) with $94 \%$ and $66 \%$ respectively and least frequently for IDV (indinavir) with $42 \%$ and $30 \%$ respectively in the analyses of current as well as previous drugs. Resistance with regard to nevirapine (NVP) was detected in all patients taking the drug; however the number was very small $(n=5)$.

FOLLOW UP OF PATIENTS WITH RESISTANCE GUIDED SWITCH OF ANTIRETROVIRAL THERAPY Individuals who were able to switch to a regimen with at least two new susceptible drugs (group 1) versus those with no or one new susceptible drug (group 2) were followed up for evaluation: 15 of 20 patients (75\%) with at least two new susceptible drugs remained on unchanged antiretroviral therapy after 24 weeks compared with six of 24 individuals $(25 \%)$ with no or one new susceptible drug. One patient per group died between week 10 (2) and week 24 (2) and two patients were lost to follow up. Two patients from the first group

Table 1 Cross resistance: distribution of individuals according to the number of drugs within one class to which their viral isolates showed $>10$-fold phenotypic or genotypic resistance. NNRTI are omitted because of insufficient data

\begin{tabular}{lllllll}
\hline & 0 & 1 & 2 & 3 & 4 & 5 \\
\hline RTI & 1 & 10 & 35 & 5 & 1 & 0 \\
PI & 13 & 11 & 4 & 7 & 17 & - \\
\hline
\end{tabular}




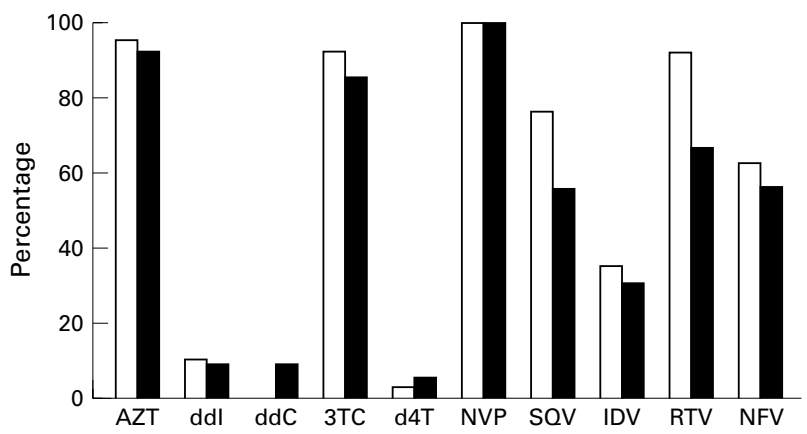

Number of patients: $19501033 \quad 0 \quad 353947 \quad 3140 \quad 5 \quad 5 \quad 1749 \quad 1427 \quad 16351316$

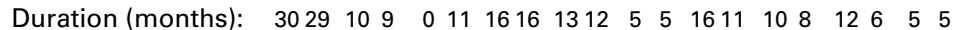

\begin{tabular}{|ll|}
\hline $\begin{array}{l}\square \text { Currently taken drug } \\
\text { (median continuous duration) }\end{array}$ & $\begin{array}{l}\text { Previously taken drug } \\
\text { (median cumulative duration) }\end{array}$
\end{tabular}

Figure 2 Isolates with $>10$-fold resistance against antiretroviral drugs of previous regimens and current regimen.

and 15 patients from the second group had to change their antiretroviral therapy. Treatment outcome in patients who were only able to add one or no new drug to their regimen was improved by the following: individuals with resistance against $\mathrm{AZT}$ and $3 \mathrm{TC}$ were most frequently switched to combinations of DDI plus D4T plus PI or non-nucleoside analogue inhibitors of reverse transcriptase (NNRTI). Therefore, 26 patients who were switched to three of these substances without documented resistance were evaluated. Among these patients, the former antiretroviral therapy in 15 patients contained two or more drugs with documented resistance and in 11 patients contained less than two such drugs. After 10 (2) weeks, viral load decrease was more pronounced in patients who had been resistant to two or more drugs of the previous regimen (fig 1B). After 24 (2) weeks the difference was less pronounced.

\section{Discussion}

Contrary to other recently published studies on HIV drug resistance-that is, VIRADAPT, GART or VIRA $3001,{ }^{4-6}$ this study only enrolled extensively pretreated individuals: all patients had received at least three different previous antiretroviral drug regimens (median $=6$ therapies). The above mentioned studies resulted in a considerable advantage of resistance guided ART switches in contrast with switches based on treatment history alone. Resistance guided switches of ART in this investigation led to a median decrease of viral load of no more than $0.5 \log 10$. This is less than the reported mean decrease of about 1 $\log 10$ in the group with resistance guided switch in the VIRADAPT study, and similar to control group results of that study. ${ }^{4}$

Data presented here suggest a striking difference in occurrence of detectable resistance to 3TC and AZT compared to DDI, D4T, and DDC. While AZT and 3TC resistant isolates were detected in over $80 \%$ of patients, resistance to DDI, DDC, and D4T was rarely detectable $(<10 \%)$ in spite of the fact that all patients had virological failure to their therapy and most of them were previously treated with DDI and/or D4T. A similar finding was reported by Shafer et al who reported four heavily pretreated patients: all patients were taking their respective antiretroviral therapy with AZT and later added or substituted other drugs over a period of 4-9 years. In all four cases high level resistance to AZT and 3TC but low level resistance against DDI, DDC, and D4T were reported. ${ }^{10}$ Two facts may be considered for explanation: AZT had been given as the first antiretroviral drug to most of our patients and duration of treatment was more than twice as long as for the other drugs.

It is conceivable that the resistance pattern against AZT developed first and persisted in the further course of infection, and further mutations eventually appearing with DDI or D4T therapy were no longer drug specific. Furthermore, it should be discussed whether the use of modified cut-off values could explain discrepancies between resistance test results and clinical success of antiretroviral therapy. Only recently new cut offs for phenotypic resistance analysis have been introduced (for example, 3.5 instead of 4.0 for DDI and DDC; 3.0 instead of 4.0 for D4T). In a recent publication, analysis of 5000 clinical samples using these cut-off values demonstrated that resistance to D4T increases by $5.4 \%$, to DDI by $3 \%$, and to DDC by $2 \% .^{11}$ Additional investigations should be performed to clarify impact and effect of new cut offs regarding response to antiretroviral therapy. Another explanation could be, that resistance to DDI, D4T, and DDC is not detectable with resistance tests used in this study. Earlier studies indicate that resistance to these substances is difficult to $\operatorname{detect}^{12}{ }^{13}$ and that mutations, commonly associated with AZT resistance, recently called TAMs or NAMs, appear during therapy with these drugs. ${ }^{14-16}$ Susceptibility to nucleosides is known to depend on inconstant levels of cellular phosphokinases, an effect that is not taken into account in resistance tests. ${ }^{17}{ }^{18}$ Additionally, it is possible that AZT and 3TC fail because resistance develops, while other RTIs fail because of adherence or for pharmacokinetic reasons. In recently performed studies at our institution we found self reported adherence not different between patients taking AZT and/or 3TC and those taking DDI, D4T, and/or DDC (data not shown). Generally, pharmacokinetic reasons are difficult to assess because therapeutic drug monitoring procedures for RTIs are not fully adequate. This is because of difficulties with the measurement of active metabolites (intracellular triphosphates), which would be the relevant measurement targets.

Resistance guided switch of therapy led to better results in patients who had resistant isolates against two or more drugs in the previous regimen, compared to those with less than two drugs, when both were switched to a regimen containing three susceptible drugs after testing (fig 1B).

Absolute reduction of the viral load was limited even in patients switched from a regimen with two or more resistant drugs to three 
susceptible drugs - that is, DDI, D4T, and a PI or NNRTI. It must be noted that subjects switched to a regimen with two or more susceptible drugs after testing remained on the same regimen considerably longer than patients who were not able to switch to a regimen with at least two new drugs. Taking all these results into account, it seems that patients will benefit most when resistance tests are performed at earlier disease stages - that is, after first failure of therapy. ${ }^{19}$ In therapy naive patients, resistance testing can detect primary resistance mutations. After failure of initial therapy, in many cases, only few resistance mutations are present and the virus population is relatively homogeneous; in these cases resistance tests offer a good chance for selecting a suppressive regimen. By increasing the number of consecutive therapy regimens and increasing the number of resistance mutations, the mutational landscape in clinical isolates becomes more complex, so does interpretation of resistance test results. Guidance taken from treatment history is highly valuable at this stage. In later stages of disease the virus has accumulated broad cross resistance to nearly all available drugs. The goal of complete suppression of viral replication may be less important at this stage; it might be more important to maintain a certain selection pressure towards less aggressive mutants.

In summary, resistance analyses seem primarily of value in first or second switch of therapy or to select a primary regimen.

The authors thank Professor L Gürtler, Greifswald, Germany, as well as $M$ van Houtte, Mechelen, Belgium, for the valuable discussion and critical review of the manuscript

Contributors: AP (principal investigator) was responsible for study design, study coordination, writing the manuscript; DA, writing the manuscript; TI, examination of patients at departwriting the of patients at department site, medical statistics, computational work; VP, data evaluation, statistics, writing and revising the manuscript, examination of patients at department site; HP, performance of scientific support; TF, coordination at laboratory site, supervision and quality controlling of genotypic testing in laboratory Fenner \& Co; TM, performance and evaluation of genotypic analyses in laboratory Arndt/Keeser; RA, coordination, supervision and quality controlling of genotypic testing in laboratory Arndt/Keeser; KH, performance of phenotypic resistance analyses at Virco laboratory; discussion of results; RP, performance of phenotypic resistance analyses at Virco laboratory; discussion of results; TW, examination of patients at department site, data evaluation, computational work; AS, responsible for study design, study coordination, subject recruitment, examination of patients.

1 Pallela FJ, Delaney KM, Moorman AC, et al. Declining morbidity and mortality among patients with advanced human immunodeviciency virus infection. $N$ Engl $7 \mathrm{Med}$ 1998;338:853-60

2 Paris D, Ledergerber B, Weber R, et al. Incidence and predictors of virologic failure of antiretroviral triple-drug therapy in a community-based cohort. AIDS Res Hum Retrovir 1999;15:1631-8.

3 Staszewski S, Miller V, Sabin C, et al. Rebound of HIV-1 viral load after suppression to very low levels. AIDS 1998; 12:2360.

4 Durant J, Clevenbergh P, Halfon P, et al. Drug-resistance genotyping in HIV-1 therapy: the VIRADAPT randomised controlled trial. Lancet 1999;353:2195-9.

5 Baxter JD, Mayers DL, Wentworth DN, et al. Final results of CPCRA 046: a pilot study of antiretroviral management based on plasma genotypic antiretroviral resistance testing (GART) in patients failing antiretroviral therapy. Antiviral Ther 1999;4 (suppl 1):6.

6 Cohen C, Hunt S, Sension M, et al. Phenotypic resistance testing significantly improves response to therapy: a randomized trial (VIRA 3001). 7th Conf Retrov Opp Inf 2000, Abstract 237.

7 Zöllner B, Feucht HH, Weitner L, et al. Drug-resistance genotyping in HIV-1 therapy. Lancet 1999;354:1120-1.

8 Hammond J, Larder BA, Schinazi RF, et al. Mutations in retroviral genes associated with drug resistance. In: Korber $\mathrm{B}$, Foley $\mathrm{B}, \mathrm{McCutch}$ an $\mathrm{F}$, et al eds. Human retroviruses and AIDS 1998. Theoretical Biology and Biophysics Group T10, Los Alamos National Laboratory, USA.

9 Hertogs K, de Bethune MP, Miller V, et al. A rapid method for simultaneous detection of phenotypic resistance to inhibitors of protease and reverse transcriptase in recombinant human immunodeficiency virus type 1 isolates from patients treated with antiretroviral drugs. Antimicrob Agents Chemother 1998;42:269-76.

10 Shafer RW, Winters MA, Palmer S, et al. Multiple concurrent reverse transcriptase and protease mutations and multidrug resistance of HIV-1 isolates from heavily treated patients. Ann Intern Med 1998;128:906-11.

11 Larder BA, Harrigan PR. Biologically relevant cut off values for phenotypic HIV-drug susceptibility testing based on the variation in drug susceptibility in untreated individuals. 3rd Europeen Symposium on the Clinical Implications of HIV Drug Resistance Frankfurt 2001, Abstract 36.

12 Miller V. Development of resistance to didanosine and stavudine in patients receiving didanosine or stavudine as part of their antiretroviral regimen. 2nd International Workshop on HIV Drug Resistance \& Treatment Strategies, 1998, Abstract 97.

13 Salomon H, Montaner JS, Belmonte A, et al. Diminished HIV-1 sensitivity to stavudine in patients on prolonged therapy occurs only at low levels and cannot be attributed to any single amino acid substitution in reverse transcriptase. Antivir Ther 1998;3:177-82.

14 Holguin A, Dietrich U, Immelman A, et al. Genotypic and phenotypic resistance to stavudine after long term monotherapy. BMS-020 Spanish Study Group. Antivir Ther 1998;3:183-6.

15 Pellegrin I, Izopet J, Reynes J, et al. Emergence of zidovudine and multidrug-resistance mutations in the HIV-1 reverse transcriptase gene in therapy-naive patients receiving transcriptase gene in therapy-naive patients receiving
stavudine plus didanosine combination therapy. AIDS 1999;13:1705-9.

16 Coakley EP, Gillis JM, Hammer SM. Phenotypic and genotypic resistance patterns of HIV-1 isolates derived from individuals treated with didanosine and stavudine. AIDS 2000;14:F9-15.

17 Friedland A, Paibir S, Srinivas M, et al. Involvement of an active effluc pump in the cellular resistance of antiretroviral nucleoside analogs. Antiviral Res 1998;37:40.

18 Moore KHP, Barrett JE, Shaw S, et al. The pharmacokinetics of lamivudine phosphorylation in peripheral blood mononuclear cells from patients infected with HIV-1. AIDS 1999;13:2239-50.

9 Boucher CAB. HIV drug resistance tests are here to stay. Curr Opin Infect Dis 1999;12:27-32. 